\title{
The Formalization Research on Self-adaptive Psychological Surveying System
}

\author{
Lan $\mathrm{Luo}^{1}$, Tianlin Chen ${ }^{2, *}$ \\ ${ }^{1}$ School of Humanity, Jiangxi University of Traditional Chinese Medicine Nanchang, 330004, China \\ ${ }^{2}$ Psychological Couseling Center, Jiangxi University of Traditional Chinese Medicine Nanchang, \\ 330004, China
}

Keywords: Computer Assist Test; Surveying; self-adaptive; Psychological Surveying System

\begin{abstract}
As a newly-developed surveying method, the Computer Assist Test (which abbreviates for CAT) can not only be widely used both in classical tests and modern surveying with its strong theoretical property and technology compatibility. Its development injects new life into surveying and effectively promotes the development of surveying theory and technology. Particularly, a branch of it-Computer Assist Test (which abbreviates for CAT) makes undeniable contributions to the development of modern surveying theory. This paper conducts the research on self-adaptive psychological surveying system by using the CAT technology, which makes users get rid of the complex calculation procedure by having no need to know the corresponding complex theory. In this way, it promotes the popularization, application and development of the modern surveying theory.
\end{abstract}

\section{Preface}

Being controlled by a particular program, the computer self-adaptive testing is a self-adaptive testing system to achieve automation and semi-automation on the computer. Theoretically, the computer self-adaptive testing can rely on different theories according to its need, but from the current application situation, the item response theory is the most widely used theory.

With a set of set molding theory, foreign Research on self-adaptive testing system started much earlier and deeper and its application range is relatively broader. In addition, other than using for psychological test, but also for some large formal examinations such as the US Graduate Record Examination, the National Nurses Commission Examination, English Qualification Examination and Certification Level of the UK.

In China, Neusoft MCEs and GRE / GMAT / TOEFL have begun to use the self-adaptive testing form. Thereinto, GRE and TOEFL are two computer self-adaptive testing systems with the broadest application and the biggest influence currently. Besides, the examining committee of National College English Test (CET- 4 and 6) has been committed to the research and development of item response theory and "the computer assist self-adaptive testing". Currently, the item response theory has been successfully used in the CET score equating processing, while the self-adaptive testing software is still under the research and construction process and hasn't have finished software model.

\section{Overall Design Concept of the System}

In order to achieve the technical advancement, extensive adaptability, manageable security and practical convenience, the overall design of the system follows the following design concept.

(1) Intelligence Management

The self-adaptive system should have a certain self-management skills that can be able to run unattended and respond to some minor problems occur daily, which requires the system to be equipped with certain intelligence. Intelligence is one of the important design concepts and objectives of this system and only intelligent system can have high stability, wide adaptability and strong vitality. 
(2) Modular Construction

As an important modern programming design concept, modular design can divide large software into small units with individual functions and can be completed by different developers simultaneously. It improves the programming development efficiency, makes the programs more convenient and efficient and accelerates the software development process. Specifically speaking, the modular design has the following advantages: l, the functions of various modules are relatively independent and can be completed jointly by different people to accelerate the software development progress. 2, the interference between the various modules makes it easy to identify and manage the problems if the system breakdowns. 3, it facilitates the administrator of the system to improve certain modules and upgrade the entire system due to the dependence of each modular.

(4) Humanization Interface

Psychological tests have stringent requirements on the the psychological state before and during the tests. Formal tests need to arrange the surrounding environment in advance, reduce noise, light, color, space size, orientation and other factors that may cause interference. Besides, there should be an instruction of the experimenters before the tests to guide the test subjects into the predetermined test situations, so as to achieve better test results.

\section{The Overall Structure of the System}

The overall structure of the system is shown in the figure 1.

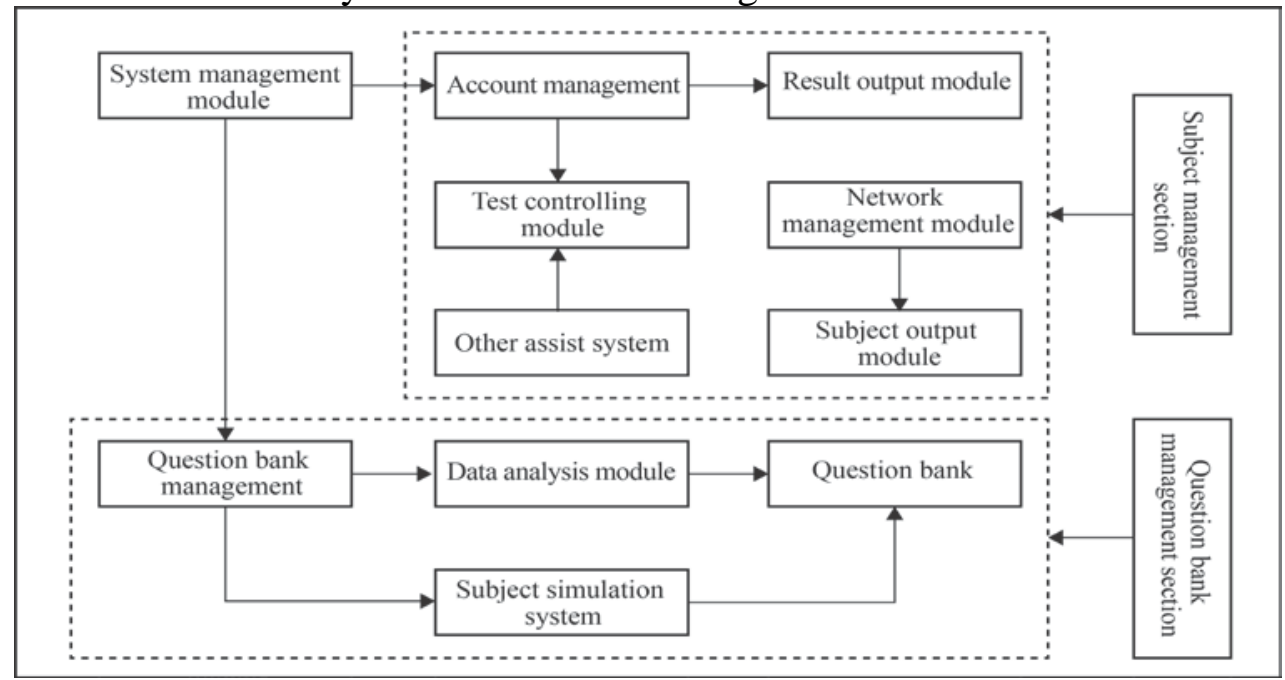

Figure 1 the overall structure of the system

(1) System Management Module

System management module is mainly responsible for the artificial management of the system, which includes account management, subject information management and the construction and management of the question bank. It is the entrance for administrators to maintain and adjust the system, anyone, including the administrators, who wants to be under the system management status should has his user name and password. Under normal circumstances, the system management module should be displayed in the program's main interface to enter through, but on special occasions, the entrance also can be hidden by the administrator in order to ensure the security of the system and prevent maliciously intrusion and vandalism.

(2) Test Controlling Module

Test is always the most important application of the system and all measures taken on the system are eventually for the test, so everything should serves for the test and all administrators should make every effort to ensure the smooth and successful implementation of the testing procedure. Before the test, subjects need to select the test to be carried out first and these tests are set beforehand by the experimenters. Subjects should complete the whole test independently under the instruction of the system. The diagrammatic sketch of the test controlling module is shown in Figure 2. 


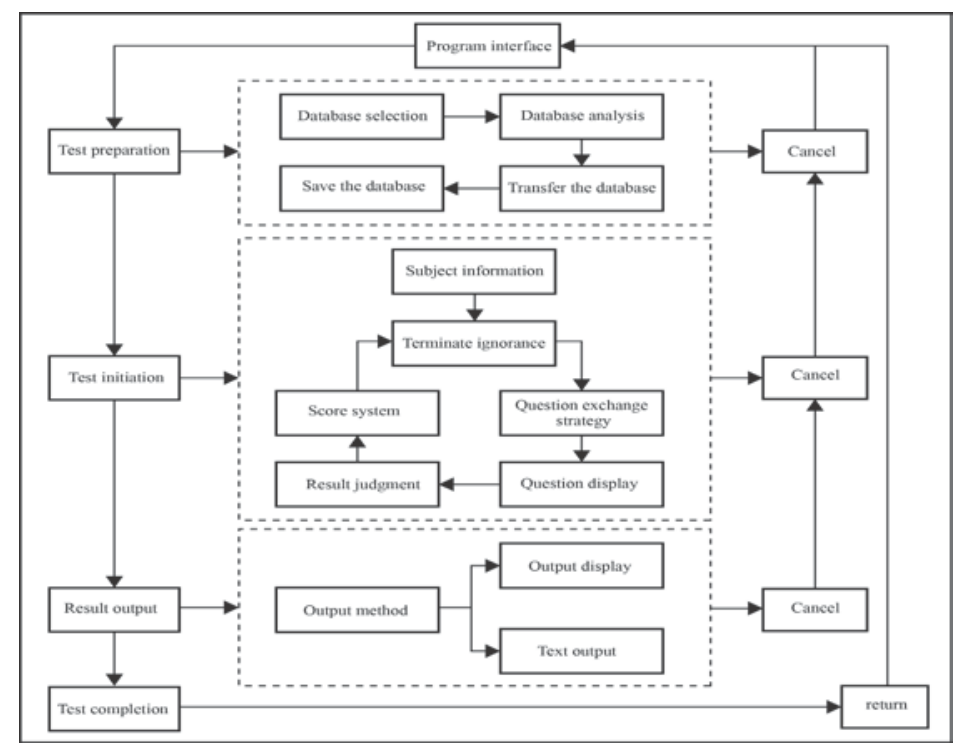

Figure 2 the diagrammatic sketch of the test controlling module

Test is always the most important application of the system and all measures taken on the system are eventually for the test, so everything should serves for the test and all administrators should make every effort to ensure the smooth and successful implementation of the testing procedure. Test scoring should be based on item response model, scores can be directly obtained through the project parameters and corresponding item response models in the case of knowing the project parameters. The specific calculation method can use Newton - Raphson iterative method (NR) and Fishersocre.

(3) Subject Input Module

The subject input module is mainly responsible for the record of answer results made by subjects, which closely connects with test controlling module in many cases, but when measured in a network administered test in order to ensure safety, we must have a certain degree of independence. The biggest difference between it and the test controlling module is that the latter is always running on the server, while the subject input module is always running on the computer for the subjects to answer. Subjects input through the network as shown in Figure 3.

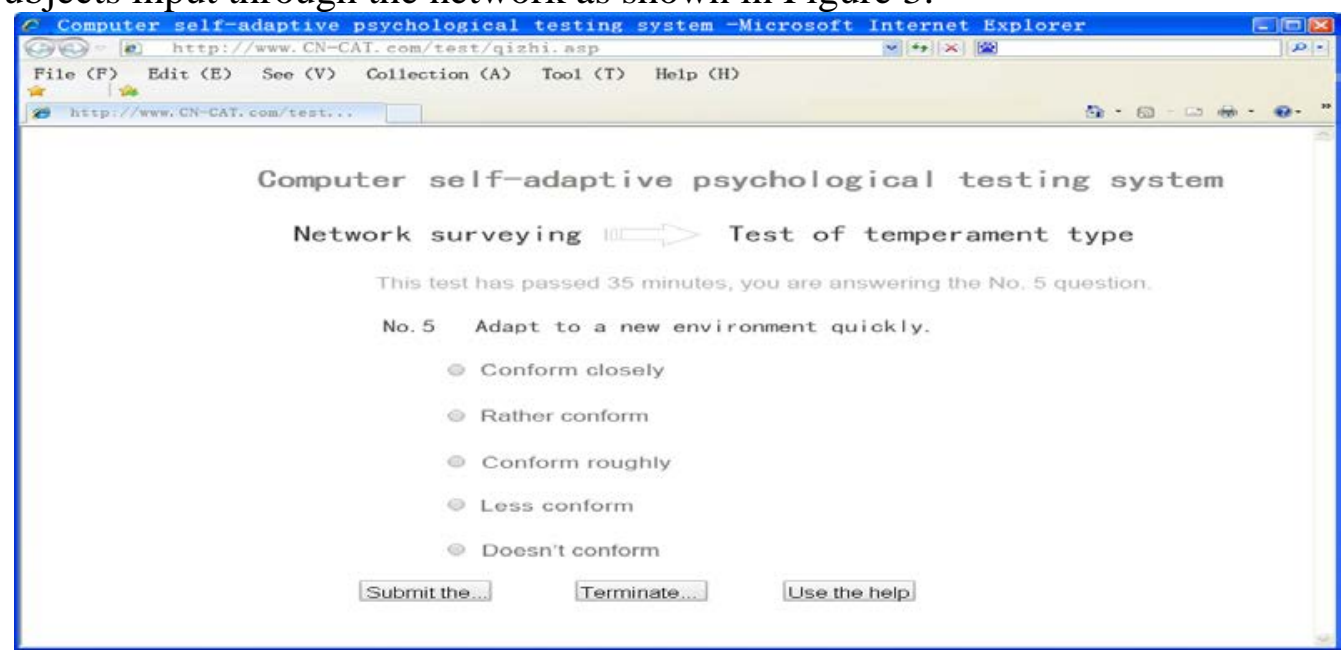

Figure 3 the diagrammatic sketch of subject network answer interface

\section{Result Output Module}

The result output is the last stage of testing process. It is completed by the experimenters, who can directly display the final results of the test on the screen or don't display the results. When finishing a certain number of test subjects, the experimenters can check the test results by using the output module of the system. The output of test results is divided into three forms: simulation paper output, data result output and team analyzed data output, which is characterized as Table 1. 
Table 1 various output forms

\begin{tabular}{|c|c|c|c|c|c|}
\hline Output form & $\begin{array}{c}\text { Original } \\
\text { dat }\end{array}$ & $\begin{array}{c}\text { Individual } \\
\text { analysis }\end{array}$ & $\begin{array}{c}\text { Team } \\
\text { analysis }\end{array}$ & Print available & $\begin{array}{c}\text { Further } \\
\text { research }\end{array}$ \\
\hline $\begin{array}{c}\text { simulation paper } \\
\text { output }\end{array}$ & Yes & Yes & No & Cannot \\
\hline Data result output & No & Yes & No & Can & Can \\
\hline $\begin{array}{c}\text { Team analyzed data } \\
\text { output }\end{array}$ & No & No & Yes & Can & Can \\
\hline
\end{tabular}

\section{System Analysis}

This system is designed on the basis of the research findings of item response theory and previous practical application. The system absorbs the essence of many previous studies and improves some of their deficiencies, so this system is improved in science, feasibility, stability and safety aspects to some extent.

Since this system is designed based on the theory, the actual stability of the system is difficult to know, but modules and functions are added to the system to enhance the stable operation of the system. Although the security is not the emphasis of the self-adaptive testing system, its significance also cannot be overlooked. Only a secure testing system can guarantee the stable operation of the test and the reliability of test result.

\section{Conclusion}

This paper is a formalization research of self-adaptive psychological testing system based on the computer technology and eight modules are designed. In addition to the basic control module and the subject input module that are needed for test, the other six modules are added modules to improve the system functionality. They add many useful functions to make it more perfect, such as system management module makes the system facilitate maintenance and management, result output module enables test results presented in various ways to conduct further research, data analysis module makes question bank management more easily, network management module, other assist systems and subject simulation system add additional auxiliary functions to allow the system more secure and feature-rich.

\section{Reference}

[1]Hmableton,R. K\&Swmainhatna H. Item response theory: Principles and Applications. Boston, MA: Kluwer-Nijho.1985.

[2]Load M.F. Application of item response theory to practical testing problem Hillsdale NJ: Erlbau, 1990.

[3] LI Hong, Che Hongsheng. The Research and Development of Computer-based Test [J]. The Dynamic Condition of the Psychology, 1999, 7(2):23-28.

[4] WANG Xijuan. CAT Research and Implementation According to the C Level [D]. Master's Thesis. Nabcahng: Jiangxi Normal University.2004.5:13.

[5] QI Qingshu. The Establishment and Application of Computer-based Self-adaptive testing [J] Jiangxi Educational Research, 1999, (2):64-66. 